\title{
Motion generates entanglement
}

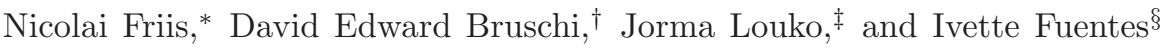 \\ School of Mathematical Sciences, University of Nottingham, \\ University Park, Nottingham NG7 2RD, United Kingdom
}

(Dated: April 2012)

\begin{abstract}
We demonstrate entanglement generation between mode pairs of a quantum field in a single, rigid cavity that moves nonuniformly in Minkowski space-time. The effect is sensitive to the initial state, the choice of the mode pair and bosonic versus fermionic statistics, and it can be stronger by orders of magnitude than the entanglement degradation between an inertial cavity and a nonuniformly moving cavity. Detailed results are given for massless scalar and spinor fields in $(1+1)$ dimensions. By the equivalence principle, the results model entanglement generation by gravitational effects.
\end{abstract}

PACS numbers: 03.67.Mn, 03.65.Yz, 04.62.+v

In the emerging field of relativistic quantum information, the past decade has revealed a number of novel kinematic effects on entanglement between observers in relative inertial motion [1] and between one inertial and one uniformly linearly accelerating observer (see Refs. [2 8 ] for a selection and Ref. 9] for a recent review). The phenomenon that has received most attention is the entanglement degradation caused by relative acceleration, assuming that the issues of switching the acceleration on and off are negligible and field modes of plane wave type, spread over large regions of the space-time, are considered. A rare case of entanglement generation under these assumptions is found in Ref. [10].

A framework that removes the idealisation of spatially unlocalised field modes is to confine the field to a cavity [1], and the idealisation of everlasting acceleration can be removed by taking the cavity's worldtube to be inertial outside a finite time interval 12]. This creates a situation that should be more directly experimentally accessible. When the cavity's worldtube consists of inertial and uniformly linearly accelerated segments, it was shown in Refs. [12, 13] that the evolution of the quantum field is amenable to a systematic perturbative analysis when the accelerations are small compared with the cavity mode frequencies, both for bosonic and fermionic fields; further, the perturbative analysis remains valid for arbitrary durations of the individual segments, and the distances travelled may hence be arbitrarily large. The nonuniformly accelerated motion was found to degrade mode entanglement between the moving cavity and an inertial reference cavity. The physical mechanism behind the degradation is that when observations of the field are restricted to a small number of field modes, for example by appealing to the frequency sensitivity profile of a detector by which the field might be observed, particle creation within the moving cavity causes information to be lost into the infinitely many field modes that are not being observed.

In this article we show, within the above perturbative treatment of a quantum field that is confined to a single cavity, that nonuniform motion of a rigid cavity creates entanglement between any two given field modes within the cavity, even when the initial state is separable. We develop a general quantitative analysis for both a scalar field and a fermion field, giving detailed results for a sample travel scenario and demonstrating that the particle statistics has a significant effect on the entanglement. As a highlight, we show that the entanglement generation can appear in the first order in the small acceleration expansion, while the entanglement degradation between cavities is only a second-order effect [12, 13]. This suggests that entanglement generation in a cavity may be more readily observable than the entanglement degradation between cavities, allowing more effective tests of phenomena linked to the dynamical Casimir effect 14], and possibly also improving the technological prospects of building quantum gates based on acceleration effects. For instance, highly entangled two-mode squeezed states, produced by a known gate in continuous variable systems, can be generated by periodically repeating segments of uniformly accelerated and inertial motion [15].

We work in $(1+1)$-dimensional Minkowski space with metric signature $(-+)$ : additional transverse dimensions can be included via their contribution to the effective field mass. The length of the cavity in its instantaneous rest frame is $\delta>0$. The cavity is assumed to be inertial outside a finite time interval, but the initial and final velocities need not coincide. We use units in which $\hbar=$ $c=1$. Complex conjugation is denoted by an asterisk and Hermitian conjugation by a dagger. $O(x)$ denotes a quantity for which $O(x) / x$ is bounded as $x \rightarrow 0$.

Bosons: We consider a real scalar field $\phi$ satisfying the Klein-Gordon equation $\left(-\square+m^{2}\right) \phi=0$, where $m \geq 0$ is the mass and $\square$ is the scalar D'Alambertian. For definiteness, we adopt at the cavity boundaries the Dirichlet boundary condition as in [12], although much of the analysis holds for any boundary condition that ensures unitarity of the time evolution.

Let $\left\{\phi_{n} \mid n=1,2, \ldots\right\}$ be a complete orthonormal set of mode solutions that are of positive frequency with re- 
spect to the cavity's proper time at early times (the inregion), and let $\left\{\tilde{\phi}_{n} \mid n=1,2, \ldots\right\}$ be a similar set at late times (the out-region). Each set has an associated set of ladder operators, with the respective commutation relations $\left[a_{n}, a_{m}^{\dagger}\right]=\delta_{n m}$ and $\left[\tilde{a}_{n}, \tilde{a}_{m}^{\dagger}\right]=\delta_{n m}$, and a vacuum state, denoted respectively by $|0\rangle$ and $|\tilde{0}\rangle$. The two sets of modes are related by the Bogoliubov transformation

$$
\tilde{\phi}_{m}=\sum_{n}\left(\alpha_{m n} \phi_{n}+\beta_{m n} \phi_{n}^{*}\right)
$$

and the ladder operators are related by

$$
a_{n}=\sum_{m}\left(\alpha_{m n} \tilde{a}_{m}+\beta_{m n}^{*} \tilde{a}_{m}^{\dagger}\right)
$$

where the notation is as in Ref. [16]. The vacua are related by 17]

$$
|0\rangle=N e^{W}|\tilde{0}\rangle
$$

where $W:=\frac{1}{2} \sum_{p q} V_{p q} \tilde{a}_{p}^{\dagger} \tilde{a}_{q}^{\dagger}, V:=-\beta^{*} \alpha^{-1}$ and $N$ is a normalisation constant.

We prepare the system in the in-region in a state without mode entanglement. We ask: does the cavity's motion generate mode entanglement in the out-region, where the particle content of the state has changed?

Our methodology is as follows. We first specify the in-region state and express it in the out-region basis using (3) and the adjoint of (2). We then trace over all out-region modes except those labelled by two distinct quantum numbers $k$ and $k^{\prime}$. We quantify the entanglement in the resulting reduced density matrix by the negativity [18 20], defined as minus the sum of the negative eigenvalues of the partial transpose. The advantages of negativity are that it is easy to compute and it interpolates between entanglement monotones that have a more direct operational interpretation [21].

To identify a parameter regime that is treatable analytically, we assume that the Bogoliubov coefficients have Maclaurin expansions in a small dimensionless parameter $h$, such that

$$
\begin{aligned}
& \alpha=\alpha^{(0)}+\alpha^{(1)}+\alpha^{(2)}+O\left(h^{3}\right), \\
& \beta=\beta^{(1)}+\beta^{(2)}+O\left(h^{3}\right),
\end{aligned}
$$

where the superscripts indicate the power of $h, \alpha^{(0)}=$ $\operatorname{diag}\left(G_{1}, G_{2}, \cdots\right)$ and each $G_{j}$ has unit magnitude. For cavity worldtubes grafted from inertial and uniformly accelerated segments, this situation arises when the acceleration at the centre of the cavity is proportional to $h / \delta$ by a numerical coefficient that may differ from segment to segment: the parameter $h$ is in this case the product of the cavity's width $\delta$ and the acceleration at the centre of the cavity [12]. We then work perturbatively in $h$. It follows that to order $h^{2}$ we have $N=1-\frac{1}{4} \sum_{p, q}\left|V_{p q}^{(1)}\right|^{2}$ and

$$
\begin{aligned}
|0\rangle & =\left(1-\frac{1}{4} \sum_{p q}\left|V_{p q}^{(1)}\right|^{2}\right)|\tilde{0}\rangle+\frac{1}{2} \sum_{p q} V_{p q} \tilde{a}_{p}^{\dagger} \tilde{a}_{q}^{\dagger}|\tilde{0}\rangle \\
& +\frac{1}{8} \sum_{p q i j} V_{p q}^{(1)} V_{i j}^{(1)} \tilde{a}_{p}^{\dagger} \tilde{a}_{q}^{\dagger} \tilde{a}_{i}^{\dagger} \tilde{a}_{j}^{\dagger}|\tilde{0}\rangle+O\left(h^{3}\right) .
\end{aligned}
$$

As a first example, we take the in-region state to be the in-vacuum $|0\rangle$. To order $h^{2}$, the partially transposed, reduced density matrix vanishes outside a $6 \times 6$ block. Among the six eigenvalues, the only possibly negative ones are

$$
\begin{aligned}
& \lambda_{4}=-\left|\beta_{k k^{\prime}}^{(1)}\right|^{2}, \\
& \lambda_{6}=f_{k \neg k^{\prime}}^{\beta}+f_{k^{\prime} \neg k}^{\beta}-\left(\left(f_{k \neg k^{\prime}}^{\beta}-f_{k^{\prime} \neg k}^{\beta}\right)^{2}+\left|V_{k k^{\prime}}\right|^{2}\right)^{1 / 2},
\end{aligned}
$$

where $f_{m \neg n}^{\beta}:=\frac{1}{2} \sum_{q \neq n}\left|\beta_{q m}^{(1)}\right|^{2}$ and $V_{k k^{\prime}}$ is kept to order $h^{2} . \quad \lambda_{4}$ arises from coherence between $|\tilde{0}\rangle$ and $\left|\tilde{1}_{k}\right\rangle\left|\tilde{1}_{k^{\prime}}\right\rangle$, while $\lambda_{6}$ arises from coherence between $|\tilde{0}\rangle$ and $\left|\tilde{2}_{k}\right\rangle\left|\tilde{2}_{k^{\prime}}\right\rangle$.

Specialising to a cavity worldtube that is grafted from inertial and uniformly-accelerated segments, we find that a qualitative difference emerges depending on the relative parity of $k$ and $k^{\prime}$. If $k$ and $k^{\prime}$ have opposite parity, the expansions given in Ref. [12] and their massive generalisations show that $\beta_{k k^{\prime}}^{(1)}$ is nonvanishing but $V_{k k^{\prime}}^{(2)}=0$. It follows that $\left|V_{k k^{\prime}}\right|^{2}=\left|\beta_{k k^{\prime}}^{(1)}\right|^{2}+O\left(h^{4}\right)$. The leading term in the negativity is then linear in $h$ and given by $\left|\beta_{k k^{\prime}}^{(1)}\right|$. If, by contrast, $k$ and $k^{\prime}$ have the same parity, we have $\beta_{k k^{\prime}}^{(1)}=0$ and $V_{k k^{\prime}}=V_{k k^{\prime}}^{(2)}+O\left(h^{3}\right)$. The leading term in the negativity comes then from $\lambda_{6}$ and is of order $h^{2}$. Sample negativity plots for both cases are shown in Fig. 1 for a massless field when the cavity undergoes a single segment of uniform acceleration.

As a second example, we take the in-region state to be $\left|1_{k}\right\rangle$, containing exactly one in-particle. Using (5) and the adjoint of (2), we find

$$
\begin{aligned}
\left|1_{k}\right\rangle= & \sum_{m}\left(\alpha_{m k}^{*}+\sum_{p} \beta_{p k}^{(1)} V_{p m}^{(1)}\right. \\
& \left.\quad-\frac{1}{4} \delta_{m k} G_{k}^{*} \sum_{p q}\left|V_{p q}^{(1)}\right|^{2}\right) \tilde{a}_{m}^{\dagger}|\tilde{0}\rangle \\
+ & \frac{1}{2} \sum_{m p q}\left(\alpha_{m k}^{*}+G_{k}^{*} \delta_{m k}\right) V_{p q} \tilde{a}_{m}^{\dagger} \tilde{a}_{p}^{\dagger} \tilde{a}_{q}^{\dagger}|\tilde{0}\rangle \\
+ & \frac{1}{8} G_{k}^{*} \sum_{p q i j} V_{p q} V_{i j} \tilde{a}_{k}^{\dagger} \tilde{a}_{p}^{\dagger} \tilde{a}_{q}^{\dagger} \tilde{a}_{i}^{\dagger} \tilde{a}_{j}^{\dagger}|\tilde{0}\rangle+O\left(h^{3}\right) .
\end{aligned}
$$

To order $h^{2}$, the partially transposed, reduced density matrix now vanishes outside an $8 \times 8$ block. Among the first five eigenvalues, the only possibly negative one is

$$
\mu_{3}=-\sqrt{3}\left|\beta_{k k^{\prime}}^{(1)}\right|^{2}
$$


which arises from coherence between $\left|\tilde{1}_{k}\right\rangle$ and $\left|\tilde{3}_{k}\right\rangle\left|\tilde{2}_{k^{\prime}}\right\rangle$. The last three eigenvalues are the roots of a cubic polynomial, analytically cumbersome for generic values of the parameters but readily amenable to numerical work.

Specialising to a cavity worldtube that is grafted from inertial and uniformly-accelerated segments, we again find a qualitative difference depending on the relative parity of $k$ and $k^{\prime}$. In particular, if $k$ and $k^{\prime}$ have opposite parity, the leading contribution to negativity comes from the eigenvalue

$$
\mu_{8}=-\sqrt{\left|\alpha_{k k^{\prime}}^{(1)}\right|^{2}+2\left|\beta_{k k^{\prime}}^{(1)}\right|^{2}}
$$

and is linear in $h$. The negativity is in this case higher than the corresponding negativity for the in-region state $|0\rangle$. Sample negativity plots are shown in Fig. 1 for a massless field when the cavity undergoes a single segment of uniform acceleration.

Fermions: We consider in the cavity a Dirac field $\psi$ with mass $m \geq 0$, with boundary conditions that ensure unitarity of the time evolution as in Ref. [13].

Let $\left\{\psi_{n} \mid n \in \mathbb{Z}\right\}$ be a complete orthonormal set of mode solutions, such that the modes $n \geq 0$ are of positive frequency and the modes with $n<0$ are of negative frequency with respect to the cavity's proper time in the in-region. We write the in-region mode expansion of the field as $\psi=\sum_{n \geq 0} \psi_{n} b_{n}+\sum_{n<0} \psi_{n} c_{n}^{\dagger}$, so that the nonvanishing anticommutators of the fermionic ladder operators for positive frequency modes and negative frequency modes are $\left\{b_{m}, b_{n}^{\dagger}\right\}=\delta_{m n}$ and $\left\{c_{m}, c_{n}^{\dagger}\right\}=\delta_{m n}$ respectively and the in-vacuum $\| 0\rangle\rangle$ satisfies $\left.\left.b_{n} \| 0\right\rangle\right\rangle=0$ and $\left.\left.c_{n} \| 0\right\rangle\right\rangle=0$. The corresponding modes, operators and states in the out-region are denoted by a tilde.

We write the Bogoliubov transformation between the two sets of modes as $\tilde{\psi}_{m}=\sum_{m} A_{m n} \psi_{n}$, where the Bogoliubov coefficients $A_{m n}$ form a unitary matrix. The relations between the two sets of ladder operators can be written in terms of the Bogoliubov coefficients by taking appropriate inner products. The vacua are related by $\left.\left.\| 0\rangle\rangle=M e^{\mathcal{W}} \| \tilde{0}\right\rangle\right\rangle$, where $\mathcal{W}=\sum_{\substack{p \gtrless 0 \\ q<0}} \mathcal{V}_{p q} \tilde{b}_{p}^{\dagger} \tilde{c}_{q}^{\dagger}, M$ is a normalisation constant, and $\mathcal{V}_{p q}$ can be expressed in terms of $A_{m n}$ [13].

We assume again that the Bogoliubov coefficients have a Maclaurin expansion, now of the form

$$
A_{m n}=A_{m n}^{(0)}+A_{m n}^{(1)}+A_{m n}^{(2)}+O\left(h^{3}\right)
$$

where $h$ is the small dimensionless parameter, $A_{m n}^{(0)}=$ $G_{m} \delta_{m n}$ (no sum) and each $G_{j}$ has unit magnitude. We then work perturbatively in $h[13]$.

We first take the in-region state to be the in-vacuum $\| 0\rangle\rangle$ and trace over all out-region modes except a mode $\kappa \geq 0$, of positive charge, and a mode $\kappa^{\prime}<0$, of negative charge. As pointed out in Ref. [22], the tracing in a (a)
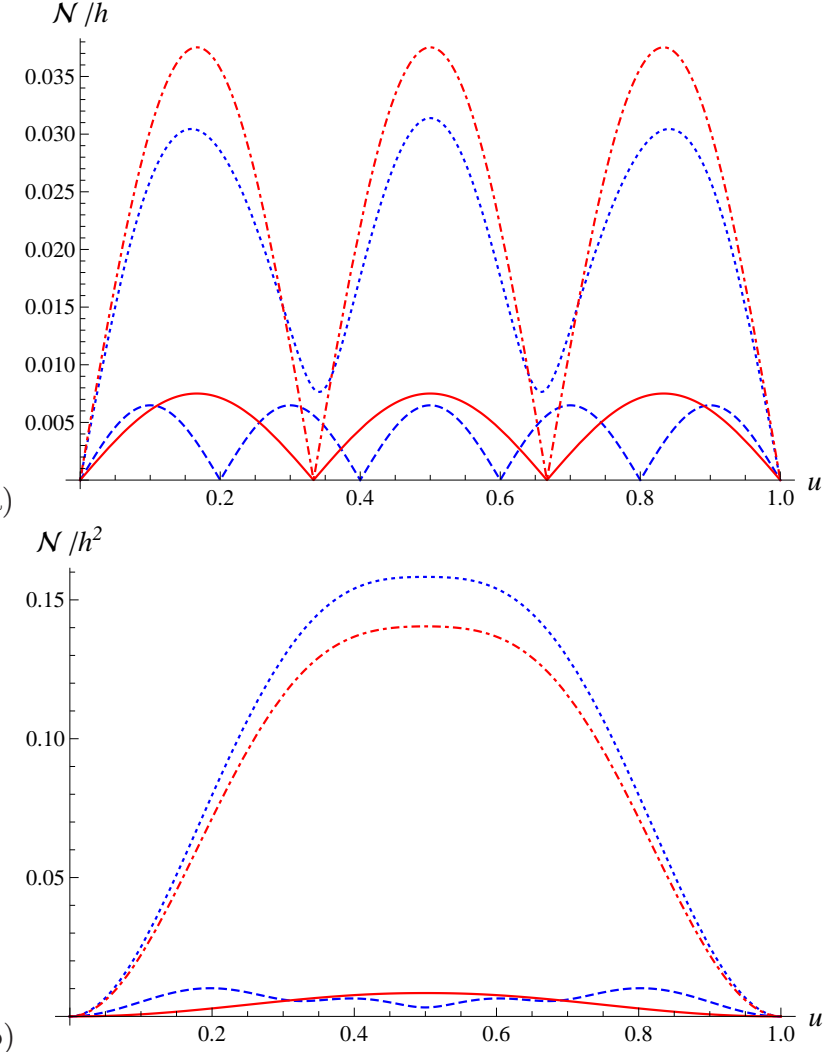

(b)

FIG. 1. The leading order contribution to the negativity is shown for a massless scalar field and a massless Dirac field. The travel scenario has a single accelerated segment, of acceleration $h / \delta$ as measured at the cavity's centre and of duration $\tau$ in the cavity's proper time, where the corresponding Bogogliubov coefficients can be found in Refs. [12, 13]. The negativity is periodic in $u:=h \tau /[4 \delta \operatorname{atanh}(h / 2)]$ with period 1 . Fig. 1(a) shows $\mathcal{N} / h$, in dashed (blue) for a scalar field with in-region vacuum and $\left(k, k^{\prime}\right)=(1,4)$, in dotted (blue) for a scalar field with in-region state $\left|1_{k}\right\rangle$ and $\left(k, k^{\prime}\right)=(1,4)$, in solid (red) for a Dirac field with in-region vacuum and $\left(\kappa, \kappa^{\prime}\right)=(2,-1)$ with $s=0$, and in dotted-dashed (red) for a Dirac field with in-region state $\left.\left.\| 1_{\kappa}\right\rangle\right\rangle$ and $(\kappa, \hat{\kappa})=(1,4)$ with $s=0$ in the notation of [13]. Fig. 1) (b) shows the corresponding curves for $\mathcal{N} / h^{2}$ with the scalar field modes $\left(k, k^{\prime}\right)=(1,3)$ and the fermionic modes $\left(\kappa, \kappa^{\prime}\right)=(1,-1)$ and $(\kappa, \hat{\kappa})=(1,3)$.

fermionic Fock space has an ambiguity, but the construction of $\mathcal{V}$ guarantees that this ambiguity does not affect our measures of entanglement, given that these measures are unambiguous for the in-region state $\| 0\rangle$. To order $h^{2}$, we find that the only potentially negative eigenvalue of the partially transposed, reduced density matrix is

$$
\nu_{3}=\bar{f}_{\kappa \neg \kappa^{\prime}}^{A}+f_{\kappa^{\prime} \neg \kappa}^{A}-\left(\left(\bar{f}_{\kappa \neg \kappa^{\prime}}^{A}-f_{\kappa^{\prime} \neg \kappa}^{A}\right)^{2}+\left|\mathcal{V}_{\kappa \kappa^{\prime}}\right|^{2}\right)^{1 / 2}
$$

where $\bar{f}_{m \neg n}^{A}:=\frac{1}{2} \sum_{\substack{q<0 \\ q \neq n}}\left|A_{q m}^{(1)}\right|^{2}$. Note the similarity with 6b.

Specialising to the massless fermion and a cavity 
worldtube that is grafted from inertial and uniformlyaccelerated segments 13], we find that the eigenvalue (11) is linear in $h$ when $\kappa$ and $\kappa^{\prime}$ have opposite parity. The leading order correction to the negativity is then equal to $\left|A_{\kappa \kappa^{\prime}}^{(1)}\right|$.

Consider then in-region states with particles. When the in-region state contains a single particle in mode $\kappa \geq 0$, the out-region reduced density matrix for two modes of opposite charge turns out to have vanishing negativity to order $h^{2}$. However, when considering two modes of the same charge as the excitation, e.g., $\kappa, \hat{\kappa} \geq 0$, entanglement is generated between these modes. Mirroring the vacuum case an analogous expression to Eq. (11), with $\bar{f}_{\kappa\urcorner \kappa^{\prime}}^{A}$ and $f_{\kappa^{\prime} \neg \kappa}^{A}$ replaced by $f_{\kappa \neg \hat{\kappa}}^{A}$ and $\bar{f}_{\hat{\kappa}}^{A}$, is obtained for the possibly negative eigenvalue of the partially transposed, reduced density matrix. Similar as before the leading order correction to the negativity is $\left|A_{\kappa \hat{\kappa}}^{(1)}\right|$ if $\kappa$ and $\hat{\kappa}$ have opposite parity.

This structure is simply a consequence of the fermionic algebra and charge conservation: entanglement is generated by the Bogoliubov coefficients connecting two modes, either by creation of a pair of oppositely charged particles in these modes, or by shifting a preexistent excitation to another mode of the same charge. If the mode in question is populated, the Pauli exclusion principle prohibits any further excitation of this mode and the creation of particle-antiparticle pairs cannot entangle it with any mode of opposite charge.

When the in-region state contains a pair of oppositely charged particles, the situation changes again: the partial transpose of the out-region reduced density matrix of the corresponding modes $\kappa \geq 0$ and $\kappa^{\prime} \leq 0$ then has one potentially negative eigenvalue of the form of Eq. (11), where $\bar{f}_{\kappa\urcorner \kappa^{\prime}}^{A}$ and $f_{\kappa^{\prime} \neg \kappa}^{A}$ are replaced by $f_{\kappa}^{A}$ and $\bar{f}_{\kappa^{\prime}}^{A}$. Specialising to the massless fermion and a cavity worldtube that is grafted from inertial and uniformly-accelerated segments [13], we find that when $\kappa$ and $\kappa^{\prime}$ have opposite parity, the leading order correction to the negativity is the same as when the in-region state is the in-region vacuum.

\section{Sample plots are shown in Fig. 1}

Conclusions: We have demonstrated that nonuniform motion of a cavity generates entanglement between modes of a quantum field confined to the cavity, both bosonic and fermionic. Working to quadratic order in the cavity's acceleration, and quantifying the entanglement by the negativity, we found that the entanglement generation depends on the initial state of the field, on the relative parity of the mode pair that is observed at late times, and on the bosonic versus fermionic statistics. For both bosons and fermions, we found situations where the entanglement generation can be enhanced by placing particles in the initial state. For fermions, however, charge conservation and the Pauli exclusion principle require the choice of the considered out-region modes to be consistent with the initial state to generate entanglement, while the bosonic statistics allow the modes to be freely populated without hindering entanglement generation.

Compared with the motion-induced entanglement degradation between a static cavity and a moving cavity [12, 13], we found that the entanglement generation can occur already in linear order in the cavity's acceleration, while the entanglement degradation is a secondorder effect. The prospects of experimental verification [12] could hence be significantly better for phenomena signalling entanglement generation than entanglement degradation.

The motion-induced entanglement effects that we have analysed have technical similarities with the creation of squeezed states in resonators with oscillating walls, known as the dynamical Casimir effect [14, 23]. In this context, we emphasise that our only approximation was to work in the small acceleration regime, meaning that the product of the cavity's length and acceleration is small compared with the speed of light squared [12, 13]. Our analysis hence covers as a special case cavities that oscillate rapidly with a small amplitude: such cavities are often introduced in theoretical analyses of the dynamical Casimir effect but are experimentally problematic 14].

Our analysis however covers also cavities that accelerate in a given direction for finite but arbitrarily long times, with travel distances that may be arbitrarily large. Further, as the equivalence principle implies that gravitational acceleration can be locally modelled by acceleration in Minkowski space-time, our results suggest that a gravitational field can produce entanglement. Experiments for entanglement generation could hence be sought in setups that span macroscopic distances, including quantum communication through near-Earth satellite orbits.

We thank Gerardo Adesso, Andrzej Dragan, Marcus Huber, Antony R. Lee and the $\chi$-QEN collaboration for helpful discussions and comments. N. F. and I. F. acknowledge support from EPSRC (CAF Grant No. EP/G00496X/2 to I. F.). J. L. was supported in part by STFC. + pmxnf@nottingham.ac.uk
$\ddagger$
$\S$ pmxdeb@nottingham.ac.uk
jorma.louko@nottingham.ac.uk
ivette.fuentes@nottingham.ac.uk previously known as
Fuentes-Guridi and Fuentes-Schuller.

[1] M. Czachor, Phys. Rev. A 55, 72-77 (1997) A. Peres, P. F. Scudo and D. R. Terno, Phys. Rev. Lett. 88230402 (2002) R. M. Gingrich and Ch. Adami, Phys. Rev. Lett. 89, 270402 (2002) D. Ahn, H. J. Lee, Y. H. Moon and S. W. Hwang, Phys. Rev. A 67, 012103 (2003) A. Peres 
and D. R. Terno, Rev. Mod. Phys. 76, No. 1, 93-123 (2004); T. F. Jordan, A. Shaji and E. C. G. Sudarshan, Phys. Rev. A 75, 022101 (2007); N. Friis, R. A. Bertlmann, M. Huber and B. C. Hiesmayr, Phys. Rev. A 81, 042114 (2010) M. Huber, N. Friis, A. Gabriel, Ch. Spengler and B. C. Hiesmayr, Euro. Phys. Lett. 95, 20002 (2011).

[2] P. M. Alsing and G. J. Milburn, Phys. Rev. Lett. 91, 180404 (2003)

[3] I. Fuentes-Schuller and R. B. Mann, Phys. Rev. Lett. 95, $120404(2005)$

[4] P. M. Alsing, I. Fuentes-Schuller, R. B. Mann and T. E. Tessier, Phys. Rev. A 74, 032326 (2006)

[5] D. E. Bruschi, J. Louko, E. Martín-Martínez, A. Dragan and I. Fuentes, Phys. Rev. A 82, 042332 (2010)

[6] E. Martín-Martínez and I. Fuentes, Phys. Rev. A 83, 052306 (2011)

[7] N. Friis, P. Köhler, E. Martín-Martínez and R. A. Bertlmann, Phys. Rev. A 84, 062111 (2011)

[8] A. Smith and R. B. Mann, e-print arXiv:1107.4633v1 [quant-ph] (2011).

[9] E. Martín-Martínez, "Relativistic Quantum Information: developments in Quantum Information in general relativistic scenarios," PhD Thesis, Complutense University of Madrid (2011) arXiv:1106.0280 [quant-ph]].

[10] M. Montero and E. Martín-Martínez, J. High Energy
Phys. 07 (2011) 006 .

[11] T. G. Downes, I. Fuentes and T. C. Ralph, Phys. Rev. Lett. 106, 210502 (2011).

[12] D. E. Bruschi, I. Fuentes and J. Louko, Phys. Rev. D 85, 061701(R) (2012)

[13] N. Friis, A. R. Lee, D. E. Bruschi and J. Louko, Phys. Rev. D 85, 025012 (2012).

[14] V. V. Dodonov, Phys. Scr. 82, 038105 (2010).

[15] D. E. Bruschi, A. Dragan, A. R. Lee, I. Fuentes and J. Louko, e-print arXiv:1201.0663 [quant-ph] (2012).

[16] N. D. Birrell and P. C. W. Davies, Quantum Fields in Curved Space (Cambridge University Press, Cambridge, England, 1982).

[17] A. Fabbri and J. Navarro-Salas, Modeling Black Hole Evaporation (Imperial College Press, London, 2005).

[18] A. Peres, Phys. Rev. Lett. 77, 1413 (1996).

[19] M. Horodecki, P. Horodecki and R. Horodecki, Phys. Lett. A 2231 (1996).

[20] G. Vidal, and R. F. Werner, Phys. Rev. A 65, 032314 $(2002)$.

[21] M. B. Plenio and S. Virmani, Quant. Inf. Comput. 7, 1 (2007).

[22] M. Montero and E. Martín-Martínez, Phys. Rev. A 83, $062323(2011)$

[23] V. V. Dodonov, A. B. Klimov and V. I. Man'ko, Phys. Lett. A 149, 225 (1990). 